\title{
Double-Needle Yamane Repositioning of a Previous Yamane Fixation
}

\author{
Sangeethabalasri Pugazhendhi ${ }^{a} \quad$ Balamurali Ambati $^{b} \quad$ Allan A. Hunter $^{c}$

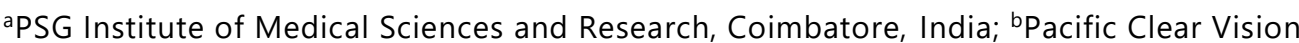 \\ Institute, Eugene, OR, USA; 'Oregon Eye Consultants, Eugene, OR, USA
}

\section{Keywords}

Yamane technique · Scleral-fixated IOLs · Double-needle intrascleral haptic fixation technique $\cdot$ Secondary IOL fixation · Pigment dispersion

\begin{abstract}
We describe a case of anteriorly dislocated, Yamane-fixated secondary intraocular lens (IOLs) with pigmentary dispersion syndrome. The patient presented with significant visual impairment and elevated intraocular pressure despite being maximally treated with all topical antihypertensive medications. The iris-IOL touch was confirmed by ultrasound biomicroscopy, and fundus examination revealed evidence of pigment granules on the optic disc. The previous Yamane-fixated secondary IOL was repositioned using a double-needle adaptation of Yamane technique and Kim's modification of scleral-fixated IOLs. To our knowledge, this is the first ever documented case of double-needle Yamane technique of a previous Yamane-fixated eye. In cases of inadequate capsular support, the development of new surgical techniques for the fixation of IOL continues to improve the safety and efficacy of these complicated surgeries.
\end{abstract}




\section{Case Reports in Ophthalmology}

\section{Introduction}

Sutureless surgical technique for secondary intraocular lens (IOL) implantation in the absence of posterior capsular support steers clear of suture-related complications. Gabor and Pavlidis [1] first documented a sutureless sulcus fixation of posterior chamber IOL by permanently fixing the haptics in a scleral tunnel parallel to the limbus. Agarwal et al. [2] reported a technique that uses biological glue in place of sutures to surgically fixate secondary IOLs in posterior capsule-deficient eyes. Other modified intrascleral IOL fixation techniques have since been reported [3-7].

The Yamane technique is a sophisticated method of IOL fixation that circumvents the need for sutures, glue, and scleral or conjunctival dissection. A modification to this technique employs 30-gauge thin-walled TSK needles allowing for a narrowed trans-scleral tunnel while ensuring adequate haptic "capture" within the needle lumen to facilitate haptic externalization [8]. The modified Yamane technique begins with keratome incision to insert an IOL into the anterior chamber. A 30-gauge TSK needle is used to create a sclerotomy incision $2 \mathrm{~mm}$ posterior to the limbus and $90^{\circ}$ from the keratome incision. The 30 -gauge TSK needle is tunneled through the sclera and into the vitreous cavity. Using intraocular forceps, the leading haptic is threaded into the lumen of the TSK needle. A second sclerotomy incision is made $180^{\circ}$ from the first sclerotomy using another 30 -gauge TSK needle, and the same steps are repeated for threading of the trailing haptic into the needle. Both needles are externalized, and an electrocautery is used to deform the haptic ends into a flange, which secures the haptics into the sclera [8].

Kim [9] described the trailing-haptic-first modification of the Yamane technique, which enhances the ease and success of the double-needle intrascleral haptic fixation technique. A superior and inferior limbal incision is created, and an IOL injector is used to insert an IOL through the superior incision. The leading haptic is grasped by microforceps as it emerges from the injector and is externalized out of the inferior incision, while simultaneously placing the trailing haptic in the anterior chamber. A 30-gauge TSK needle is used to pierce the conjunctiva and sclera to enter into the vitreous cavity. The trailing haptic is threaded into a TSK needle. Similarly, another 30 -gauge needle is placed $180^{\circ}$ from the first needle and pierced into the vitreous space. Microforceps are used to grasp the leading haptic to thread it into the lumen of the needle. Both needles are externalized to expose the haptics, which are then deformed into a flange by using an electrocautery. The bulbs are then pushed into the sclera [9]. Our case is a double-needle modified Yamane fixation of a previous Yamane technique-fixated secondary IOL.

\section{Case Report}

A 60-year-old male patient presented in October 2018 with an anteriorly Yamane-fixated IOL OS by an outside ophthalmologist and significant visual impairment. The patient had a previously placed Yamane-fixated IOL, which was rubbing against the iris causing inflammation and pigment dispersion (without macular edema) despite the presence of a patent iridotomy placed at the original surgery (Fig. 1a). The iris-IOL touch was confirmed by exam and ultrasound biomicroscopy (UBM), as shown in Figure 1b. The flanged-haptics position was measured at $1.7 \mathrm{~mm}$ (posterior from the limbus) at the $12: 00$ and $1.5 \mathrm{~mm}$ (posterior from the limbus) at the 6:00 position, respectively. This position of the flanged-haptics is anterior to the suggested $2 \mathrm{~mm}$ position posterior to the limbus as described by Yamane et al. [8]. 
Patient's best-corrected visual acuity in October 2018 was $20 / 200$, which was probably due to the poor positioning of the IOL, and his intraocular pressure was $37 \mathrm{~mm} \mathrm{Hg}$ despite being maximally treated with all topical anti-hypertensive medications. The patient's ocular hypertension and ocular condition required a repositioning of the IOL.

\section{Surgical Technique}

Once the eye is prepped, the surgeon is placed at patient's 12:00 position. A 25- or 27gauge vitrectomy setup is placed per protocol. The 25 -gauge system was used in this case for its more stable instrumentation in IOL manipulation. The infusion port is placed at 8:30 position OS (or 3:30 OD) to ensure maximum distance from the 6:00 and 3:00 position. Alternatively, the infusion ports can be placed inferonasally. Trocars are placed at 10:30 and 2:30 positions, and complete vitrectomy is performed to confirm that no residual anterior hyaloid material is within the IOL/iris plane.

The surgeon now moves to the temporal position. Peritomies of one clock hour are made at 6:00 and 12:00 positions where the haptic ends are visualized (Fig. 2a), and all tenons capsule are removed from the peritomy sites. In accordance with Kim, a new $30 \mathrm{~g}$ thin walled TSK needle is placed, $2.5 \mathrm{~mm}$ posterior from the limbus at the $6: 00$ position in a beveled manner. As this is the patient's native IOL (Zeiss CT Lucia 602), the haptic is pulled externally by an IOL grasper at the 6:00 position (Fig. 2b) and its end is cut flush with IOL cutting scissors immediately in front of the flanged haptic tip. The surgeon places 25 -gauge serrated grasping forceps to internalize the haptic and to thread the haptic into a new 30-gauge TSK needle that is retropositioned at precisely $2.5 \mathrm{~mm}$ from the limbus at the identical 6:00 position. The bevel of the needle is used as a platform to secure the haptic into the needle, as mentioned in Kim's modification. The needle is then externalized, which exposes the haptic. The tip of the haptic is deformed into a terminal bulb by electrocautery and repositioned into the sclera at the 6:00 position, as shown in Figure $2 \mathrm{c}$ and $\mathrm{d}$. The same steps are performed at the 12:00 sclerotomy (marked by arrow), as seen in Figure $3 a$ and $b$. Both peritomies are closed and infusions are removed as per protocol.

\section{Postoperative Findings}

In November 2018, the patient underwent a Yamane posterior repositioning of a Yamane technique-placed scleral-fixated IOL. The postoperative period was uneventful. Postoperative UBM was performed in December 2018, which revealed a well-fixated IOL and absence of the iris-IOL touch (image not included). The patient's visual acuity improved to better than 20/40, and there was reduction of intraocular pressure to within normal limits with a resolution of anterior chamber pigment dispersion by the third-month postoperative exam. To our knowledge, this is the first report of this surgical technique of a Yamane repositioning of a previously placed Yamane-fixated IOL.

\section{Discussion}

This case of iris-IOL touch interestingly occurred in an eye with an intrascleral-fixated IOL. The chaffing of the lens on the iris results in pigment dispersion, ocular inflammation, and elevated intraocular pressures which is resistant to maximum topical therapy. Yamane et al. [8] reported the need for peripheral iridectomy to prevent iris capture, which was the major complication in the study affecting $8 \%$ (8 eyes) of patients. The prophylactic iridectomy reduces the occurrence of iris capture caused by an intraoperative reverse pupillary block. 
Studies advise the use of laser peripheral iridotomy or surgical peripheral iridectomy to prevent iris capture in cases of reverse pupillary block $[10,11]$. This patient had a patent surgical iridotomy placed at the original IOL fixation (photo not provided). Figure 1a shows evidence of pigment granules on the disc seen after the vitrectomy due to the excessive pigment dispersion in this case. The haptics were moved $2.5 \mathrm{~mm}$ posterior to the limbus (rather than $2.0 \mathrm{~mm}$ ) in this case to allow for adequate posterior positioning of the IOL to remove all possible irisIOL touch. The positioning of the haptics was measured with calibers highlighting the importance of exact measurements and instrumentation during this type of surgery. To our knowledge, this is the first ever documented case of double-needle Yamane technique of a previous Yamane-fixated eye.

The techniques of this surgery incorporate the original double needle technique of threading and externalizing both haptics to center and fixate the IOL. The surgery also integrates Kim's modification in regard to placement of the new 30-gauge TSK needle and threading of the haptic. The 6:00 haptic was approached first followed by the 12:00 haptic in this case. However, the same surgical steps could be performed vice versa, in approaching the 12:00 haptic first before 6:00 haptic, obviously, as there is no difference in the manipulation of the haptics.

In addition to surgical technique, the lens material and surgical instruments used are equally crucial in developing an effective and easier method of secondary IOL implantation for novice and experienced surgeons. The lens used in this case is a Zeiss CT Lucia 602, made of sturdy polyvinylidene fluoride. This resilient material poses some challenges in internalizing the haptics into the lumen of the TSK needle, and surgical aplomb in the Yamane technique is suggested prior to attempting this repositioning.

The use of 30-gauge TSK in this case highlights the importance of verifying that the haptic precisely fits into the lumen of the needle to facilitate intra-lumen "capture": adequate haptic stabilization to facilitate haptic externalization.

Scleral fixation of IOLs is an effective surgical option for conditions such as IOL dislocation, aphakia, or trauma to the eye [12-14]. In the absence of adequate capsular support, surgical implantation and artificial fixation of the IOL via varied surgical techniques have gained wide popularity. The proposed technique elaborates on a simple surgical modification of the Yamane technique, which displays significant visual improvement with minimal or no intraand postoperative complications. The development of new surgical techniques continues to improve the safety and effectiveness of these complicated cases. Further studies with more patients and long follow-up periods will more accurately demonstrate structural and functional results. Despite the progress made in current surgical techniques, minor alterations can result in drastic effects on the ease and efficacy of the surgical technique for surgeons as well as substantial changes in the visual outcomes of the patients.

\section{Statement of Ethics}

The authors have no ethical conflicts to disclose. Written informed consent was obtained from the patient.

\section{Disclosure Statement}

The authors have no conflicts of interest to declare. 


\section{Funding Sources}

None.

\section{Author Contributions}

All authors are accountable for all aspects of the work and contributed to the design, analysis, and interpretation of the case data, in addition to the drafting and revision of the paper, as well as providing the final approval of the version to be published.

\section{References}

1 Gabor SG, Pavlidis MM. Sutureless intrascleral posterior chamber intraocular lens fixation. J Cataract Refract Surg. 2007 Nov;33(11):1851-4.

2 Agarwal A, Kumar DA, Jacob S, Baid C, Agarwal A, Srinivasan S. Fibrin glue-assisted sutureless posterior chamber intraocular lens implantation in eyes with deficient posterior capsules. J Cataract Refract Surg. 2008 Sep;34(9):1433-8.

3 Totan Y, Karadag R. Trocar-assisted sutureless intrascleral posterior chamber foldable intra-ocular lens fixation. Eye (Lond). 2012 Jun;26(6):788-91.

4 Ohta T, Toshida H, Murakami A. Simplified and safe method of sutureless intrascleral posterior chamber intraocular lens fixation: $y$-fixation technique. J Cataract Refract Surg. 2014 Jan;40(1):2-7.

5 Takayama K, Akimoto M, Taguchi H, Nakagawa S, Hiroi K. Transconjunctival sutureless intrascleral intraocular lens fixation using intrascleral tunnels guided with catheter and 30 -gauge needles. $\mathrm{Br} \mathrm{J}$ Ophthalmol. 2015 Nov;99(11):1457-9.

6 Rodríguez-Agirretxe I, Acera-Osa A, Ubeda-Erviti M. Needle-guided intrascleral fixation of posterior chamber intraocular lens for aphakia correction. J Cataract Refract Surg. 2009 Dec;35(12):2051-3.

7 Yamane S, Inoue M, Arakawa A, Kadonosono K. Sutureless 27-gauge needle-guided intrascleral intraocular lens implantation with lamellar scleral dissection. Ophthalmology. 2014 Jan;121(1):61-6.

8 Yamane S, Sato S, Maruyama-Inoue M, Kadonosono K. Flanged Intrascleral Intraocular Lens Fixation with Double-Needle Technique. Ophthalmology. 2017 Aug;124(8):1136-42.

9 Kim DB. Trailing-haptic-first modification of double-needle intrascleral haptic fixation technique. J Cataract Refract Surg. 2018 Apr;44(4):424-8.

10 Choi SR, Jeon JH, Kang JW, Heo JW. Risk factors for and management of pupillary intraocular lens capture after intraocular lens transscleral fixation. J Cataract Refract Surg. 2017 Dec;43(12):1557-62.

11 Higashide T, Shimizu F, Nishimura A, Sugiyama K. Anterior segment optical coherence tomography findings of reverse pupillary block after scleral-fixated sutured posterior chamber intraocular lens implantation. Cataract Refract Surg. 2009 Sep;35(9):1540-7.

12 Abbey AM, Hussain RM, Shah AR, Faia LJ, Wolfe JD, Williams GA. Sutureless scleral fixation of intraocular lenses: outcomes of two approaches. The 2014 Yasuo Tano Memorial Lecture. Graefes Arch Clin Exp Ophthalmol. 2015 Jan;253(1):1-5.

13 Sindal MD, Nakhwa CP, Sengupta S. Comparison of sutured versus sutureless scleral-fixated intraocular lenses. J Cataract Refract Surg. 2016 Jan;42(1):27-34.

14 Kelkar AS, Fogla R, Kelkar J, Kothari AA, Mehta H, Amoaku W. Sutureless 27-gauge needle-assisted transconjunctival intrascleral intraocular lens fixation: initial experience. Indian J Ophthalmol. 2017 Dec;65(12):1450-3. 


\section{Case Reports in Ophthalmology}

Pugazhendhi et al.: Double-Needle Yamane Repositioning of a Previous Yamane Fixation
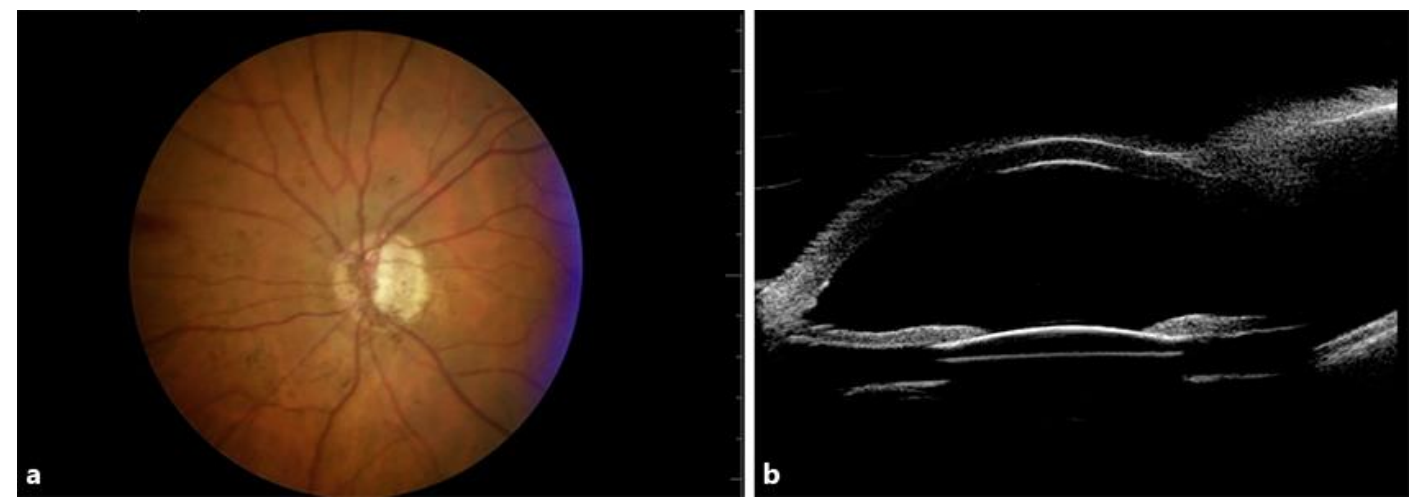

Fig. 1. a Pigmentary granules on optic disc. b Ultrasound biomicroscopy confirming iris-IOL touch.
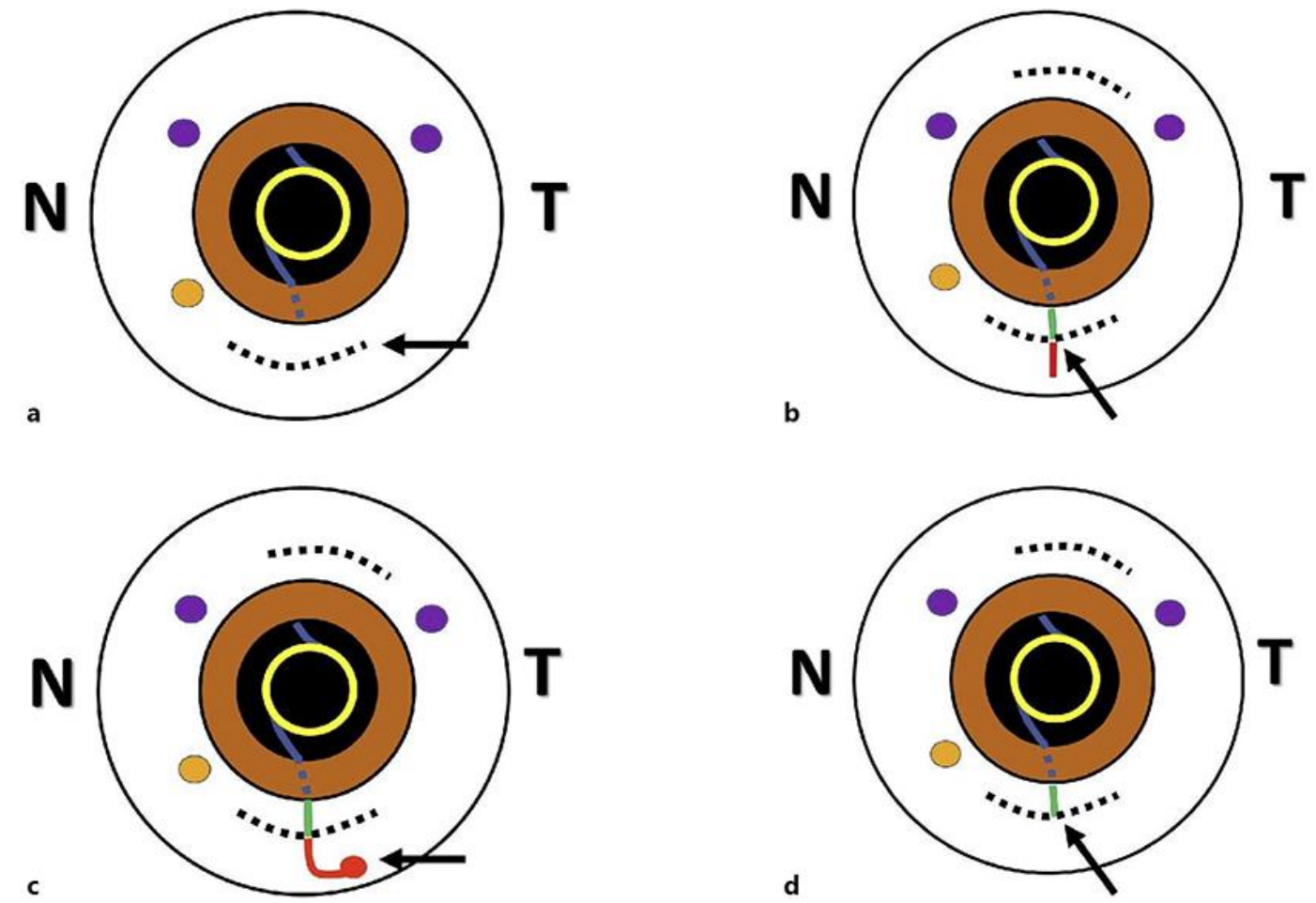

Fig. 2. a Trocar positions (purple circles) at 10:30 and 2:30 and infusion port (orange circle) at 8:30 position. Peritomy at 6:00 is marked by the arrow. b Haptic is externalized through the sclerotomy. Red haptic indicates external position, green indicates intrascleral, and blue indicates intraocular position. c Terminal flange is indicated by arrow. $\mathbf{d}$ Flange is buried trans-sclerally and peritomy closed. 

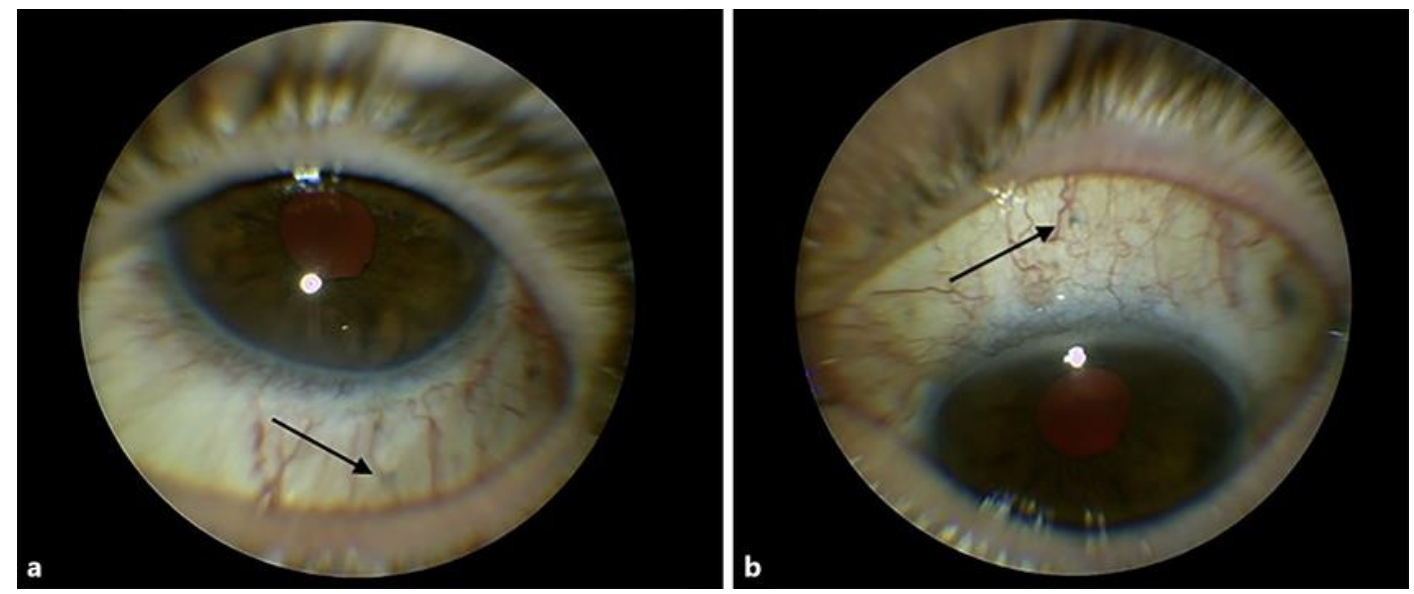

Fig. 3. a Terminal flange at 6:00 position. $\mathbf{b}$ Terminal flange at 12:00 position. 